\section{TCRs go it alone}

T cell antigen receptor (TCR) must distinguish between abundant irrelevant peptide and antigenic peptide bound to major histocompatibility complex (MHC) molecules. In eLife, Groves and colleagues use live TCR-transgenic T cells on a supported lipid bilayer to image the triggering of individual TCR-peptideMHC complexes. Triggering is determined by recruitment of the signaling molecule Zap70 to TCRs. Free peptide-MHC moves rapidly and randomly, but after engagement of the TCR, the movement becomes slow and linear, consistent with cytoskeletal involvement. Single TCR-peptide-MHC conjugates are stable, lasting up to tens of seconds, and bind at a stoichiometry of $1: 1$, yet are still able to recruit Zap70. This suggests that clustering or multimerization of receptors is not a requirement for TCR triggering. The stability of individual conjugates and the 1:1 stoichiometry therefore indicate that signal amplification must take place after TCR triggering. ZF eLife (3 July 2013) doi:10.7554/eLife.00778

\section{Networks for costimulation}

Costimulation through the coreceptor CD28 is required for efficient activation of naive $\mathrm{T}$ cells. In the Journal of Experimental Medicine, Bluestone and colleagues use gene-expression microarray to identify genes uniquely regulated by CD28 signaling in naive $\mathrm{CD} 4^{+} \mathrm{T}$ cells. DEC1, a basic helix-loop-helix transcription factor, is induced by CD28 costimulation early during $\mathrm{T}$ cell activation. DEC1-deficient $\mathrm{T}$ cells have diminished proliferation and survival after activation, which correlates with lower interleukin 2 (IL-2) production. DEC1-deficient mice are resistant to the induction of experimental autoimmune encephalomyelitis because of defects in $\mathrm{T}$ cell proliferation and cytokine production. IL-2 can restore proliferation but cannot correct an intrinsic survival defect in antigen-specific DEC1-deficient T cells. DEC1-dependent gene signatures indicate DEC1 controls the transcriptional regulation of several T cell-activation pathways, a big subset of which are also regulated by CD28.

J. Exp. Med (22 July 2013) doi:10.1084/jem.20122387

\section{Interferon-induced necroptosis}

Activation of death receptors can induce intrinsic cell death through formation of the 'necrosome' kinase complex composed of RIP1 and RIP3. However, this process is tightly regulated by FADD, caspase 8 and C-FLIP. In the Proceedings of the National Academy of Science, Thapa et al. describe an interferon-induced necroptosis pathway that is independent of signaling via death receptors. Both interferon- $\alpha / \beta$ and interferon- $\gamma$ trigger upregulation of the double-stranded RNA-dependent protein kinase PKR, which interacts with RIP1 and is necessary for formation of the RIP1-RIP3 'necrosome'. Inhibition of either interferon signaling or PKR diminishes necroptosis. Notably, necroptosis occurs only when FADD is disabled by phosphorylation of Ser191, which occurs during the G2-M stage of the cell cycle. This induced death pathway might be a failsafe mode of innate immunity to control infection by viruses or bacteria that express apoptosisinhibitor proteins.

Proc. Natl. Acad. Sci. USA (29 July 2013) doi:10.1073/

pnas. 1301218110

\section{Regulatory fraction}

The gut microbiota can be manipulated for therapeutic purposes in inflammatory and allergic diseases. In Nature, Honda and colleagues isolate and identify bacterial strains from the normal human gut that can induce regulatory $T$ cells $\left(T_{\text {reg }}\right.$ cells) in the colon of germ-free mice after inoculation. A mixture of 17 Clostridium species of clusters XIVa, IV and XVIII, which lack genes encoding strong virulence factors, acts synergistically as a bacterial community to increase the frequency of $T_{\text {reg }}$ cells in the colon of colonized mice and rats. The 17-strain mixture provides short-chain fatty acids, bacterial antigens and probably other factors to induce the differentiation and population expansion of functional colonic $\mathrm{T}_{\text {reg }}$ cells. Administration of the 17-strain mixture has a protective effect in mouse models of allergic diarrhea and experimental colitis, whereas patients with ulcerative colitis have a lower frequency of 5 of the 17 Clostridium strains.

Nature (10 July 2013) doi:10.1038/nature12331

\section{Clustering NK receptors}

Natural killer (NK) cell activity is governed by the opposing actions of activating and inhibitory receptors at the immunological synapse. In Science Signaling, Pageon et al. use super-resolution fluorescence microscopy to observe changes in the nanoscale organization of the inhibitory receptor KIR2DL1 after receptor ligation. Resting NK cells have discrete nanoclusters of KIR2DL1 that after ligation become denser aggregates. Surprisingly, ligation of the activating receptor NKG2D also triggers tighter clustering of KIR2DL1 but not of other surface molecules such as HLA, which indicates crosstalk between the two NK receptors. Unlike ligation of KIR2DL1, NKG2D-induced receptor clustering requires remodeling of the actin cytoskeleton. KIR2DL1 remodeling could lower the threshold required for transmission of its inhibitory signals via the phosphatase SHP-1. Whether this remodeling increases the proximity between KIR2DL1 and NKG2D or other downstream signaling molecules remains unknown.

Sci. Signal. (23 July 2013) doi:10.1126/scisignal.2003947

$L A D$

Written by Laurie A. Dempsey, Zoltan Fehervari \& Ioana Visan 